\title{
Quantum Paradigms of Psychopathology: From Consciousness to Neuroethics
}

\author{
Massimo Cocchi',2, Lucio Tonello ${ }^{1}$, Fabio Gabrielli1 \\ 1"Paolo Sotgiu" Institute for Research in Quantitative \& Quantum Psychiatry \& Cardiology, LUdeS, Lugano, Switzerland \\ ${ }^{2}$ Department of Veterinary Medical Sciences, University of Bologna, Bologna, Italy \\ Email: massimo.cocchi@unibo.it
}

How to cite this paper: Cocchi, M., Tonello, L., \& Gabrielli, F. (2017). Quantum Paradigms of Psychopathology: From Consciousness to Neuroethics. Open Journal of Depression, 6, 24-30.

https://doi.org/10.4236/ojd.2017.61003

Received: January 1, 2017

Accepted: February 21, 2017

Published: February 24, 2017

Copyright (C) 2017 by authors and Scientific Research Publishing Inc. This work is licensed under the Creative Commons Attribution International License (CC BY 4.0).

http://creativecommons.org/licenses/by/4.0/

\section{Open Access}

\begin{abstract}
The present work deals with the complexity of the arguments underlying the meaning of the quantum paradigm of psychopathology. In particular, the quantum approach to the understanding of the brain and consciousness, seems to present convergence of thinking of many scientists and also seems to be the most promising way in the approach of future research. The practical potency of classical neuroscience directed toward either beneficial or perverse purposes will prove in the end limited by that orthodox paradigm's inherently poor explanatory power in linking mind and brain, especially at the basic level of the Hard Problem as Chalmers (1995) has termed. However, if quantum neurobiology should demonstrate greater explanatory power than does its classical counterpart, then an enhanced potential not only for constructive psychiatric application but also for politically motivated abuse will follow with a vengeance. If the mechanistic-reductionist cognitive approaches have been characterised by the metaphor of the "edifice", of the solid Cartesian rock, all the forms of knowledge founded on complexity theory, have been characterised by the metaphor of the "network", of thinking in relationships, in a dynamic, fluid, open manner. In the field of mental illness, this means setting aside both the organicist paradigm and the pseudo-phenomenological, "sentimental", and therefore ideological, paradigm, in order to have an integrated view of biological objectiveness and humanistic psychotherapy. That is to say, an expression of diverse interrelated contributions from the various disciplines (psychiatry, psychology, biochemistry, anthropology, quantum physics, mathematics, philosophy). The observer thus becomes a builder of models, a manager of complexity, giving treatment the character of a truly empathic relationship. This is all the more so where distressing pathologies are involved, such as Major Depression (MD) and Bipolar Disorder (BD), caput mortuum of psychiatry, because the absence of cogent biological markers seriously compromises every form of therapy.
\end{abstract}




\section{Keywords}

Psychopathology, Consciousness, Quantum Consciousness, Man and Animal Consciousness, Neuroethics

\section{Introduction}

The Research Institute "Paolo Sotgiu", created in 2009 at the initiative of the biochemistry Massimo Cocchi, the biomathematics Lucio Tonello, the philosopher Fabio Gabrielli, in collaboration with the Quantum Paradigms of Psychopathology (“QPP”:

http://www.neuroquantology.com/index.php/journal/announcement/view11), born in 2008, at the initiative of Donald Mender, psychiatrist at Yale. He tried to approach the psychopathology with a multidisciplinary and interdisciplinary approach, with a strong emphasis on sciences complexity, highlighting, in particular, the contributions of philosophy and quantum physics to the signification and the diagnosis of Mood Disorders.

About quantum physics, we will say in the following lines, as regards the philosophy, two are the most relevant aspects:

1. In the light of biomolecular contiguity between man and animal, you wondered how fragile is the definition of the subject in an anthropocentric perspective. In other words, we decided to deconstruct the concept of the subject as a prerogative of the rational animal, of the man as the sole holder of subjectivity, as provided, in prime measure, of logical-rational capacities (Derrida \& Wills, 2002).

2. From the renewed interest in meta-ethics, replaced after the sixties by the ethics legislation and then, from the one applied, i.e. on the basis of the semantic elements of the moral metaphysical and epistemological assumptions, fruitful attempts of synthesis among moral philosophy, biology, psychology and, above all, neurosciences, without naturalizing indefinitely the ethical concept, are achieved (Flanagan, 1991). In particular, neuroethics, born from the dialogue between philosophy speculation and scientific research, there seems to be an unavoidable field of research for anyone involved in consciousness and psychopathology: the neurobiological foundations of morality to the experimental protocols, up to the possible ideological-manipulative tendencies of new technologies.

The following documents, even in their necessary synthesis, intend to develop some of these considerations, with particular reference to quantum approaches to consciousness and its pathological drifts.

\section{The Palermo Declaration}

On this day of April 27, 2013 a core international group of investigators (Aramini, Bernroider, Cocchi, Gabrielli, Globus, Malik, Mender, Mullis, Pessa, Pregnolato, Pylkkanen, Rasenick, Tonello, Tuszynski, Vitiello, Werneke, Zizzi), with 
expertise in the fields of psychiatry, biochemistry, physics, computational neuroscience, mathematics, philosophy and theology, gathered in Palermo, Sicily, to assess the potential relevance of quantum physics and quantum chemistry in augmenting the assumptions of non-quantum biophysics and biochemistry aimed toward understanding mind-brain relations in normal and abnormal states of consciousness applicable to humans and non-human animals.

It can be stated that:

Recent progress of a restricted kind in mainstream consciousness research has proceeded rapidly due to dramatic technical improvements in relevant empirical research tools. Classical biophysics, which provides the paradigmatic foundation of mainstream consciousness research, has offered bountiful correlations between subjective reports of qualitative human experience and quantitative measurements of objective physical processes. However, these merely correlative advances have not at all addressed what Chalmers (1995) has termed the "Hard Problem" of mind-brain relations by bridging what Levine (1983) has called the "Explanatory Gap" between qualitatively subjective phenomenal experience and quantifiably objective physical events. So far, no explanatory bridge between consciousness and corporeal neural tissue has issued from the classical biophysics of mind and brain in homo sapiens, and, in research on non-human subjects precluding self-reports via human language abilities, even correlations have remained substantially elusive. Quantum approaches may offer greater latitude in addressing these classical deficiencies, to the extent that at least some latent links formally exist between the qualitative dimensionality and quantitative measurability of canonically conjugate quantum observables, whereas no such formal links are required with reference to the possessed observables of classical physics. Moreover, at least one interpretation of quantum measurement as formulated by von Neumann (1932) casts the measuring agency itself as subjectively conscious per se, in contrast to an absence of any such classical notion.

Quantum generalization of classical biophysics opens up the possibility that relevant brain processes may reach both beneath the scale and beyond the boundaries of discrete neurons and the synaptic connections among those classically cellular "nodes". Quantum-germane structures and dynamics within the brain may include superposed dimeric tubulin conformations in the microtubular cytoskeleton spanning both intraneuronal and interneuronal spaces, ordered water in relation to cytoskeletal proteins, membrane channels and lipids along with their second messenger pipe lines to neuronal interstices, and solitons communicating along cytoskeletal routes between classical and quantum aspects of brain function. Tegmark's (2000) objections to the thermodynamic feasibility of such quantum structures and processes surviving thermal decoherence at biological temperatures entailing orders of magnitude comparable to those within the human skull have been thrown into doubt by the recent work of Gregory Engel's group, which demonstrated non-trivial quantum computation in photosynthesis. The ubiquity of water, cytoskeletal tubulin, membrane lipids, and second messengers in non-human life suggests that a new biophysics accounting for quantum-generalized processes in living tissue may lead to future predictions 
about consciousness not only in human beings but also in organisms lacking any semblance of human brain architecture at the level of organized neuronal networks or "higher."

Pothos \& Busemeyer (2009) have presented abundant empirical evidence that properties of normal mental life may be more parsimoniously modeled by abstract quantum formalisms than by classical computational algorithms. The quantum wetware outlined above is more compatible with these formalistic findings than is any classical model of neural biophysics. Both quantum-logical and quantum-physical approaches to mind and brain also promise to generate avenues for better comprehension of neurophysics in psychopathology. Explanatory and even psychotherapeutic opportunities may emerge from considerations of superpositional logic and malattunement in the primary process thinking of schizophrenia, Everett's quantum ontology in the "alternate worlds" of psychotic perception, and membrane and second-messenger interfaces between serotonin biochemistry and quantum-microtubular nanowire dysfunction in mood disorders. Aberrations of scale emergence in quantum thermofield phase transitions and problematic barriers to Bohmian holism (Bohm, 1951) may be important in multiple forms of mental illness.

We declare the following: "The absence of neurons and their synaptic connections does not preclude the presence of at least rudimentary phenomenal experience in organisms endowed with superposed microtubular dimers, ordered water, membrane ion channels, andlor crucial lipid raft assemblies connected to selected second messenger systems. In addition, quantum-biophysical aspects of these andlor other yet undiscovered structures and related processes may prove to be potent factors in the deeper etiologies and improved treatments of psychiatric disorders."

\section{The Document of Bologna}

On this day of June 20, 2014 a core international group of investigators (Bernroider, Cocchi, Gabrielli, Globus, Malik, Mender, Mullis, Pessa, Pregnolato, Pylkkanen, Rasenick, Tonello, Tuszynski, Vitiello, Werneke, Zizzi), with expertise in the fields of psychiatry, biochemistry, physics, computational neuroscience, mathematics, philosophy and theology, gathered in Bologna, Emilia Romagna, to assess the potential relevance of quantum and quantitative aspects in augmenting the knowledge and understanding of mind-brain relations in psychopathologic conditions applicable to humans.

In the light of diagnostic possibilities that these disciplines will give to the subjectivity of the psychiatric interpretation, it seems inevitable the rise of ethics aspects.

It can be stated that so far, no explanatory bridge between consciousness and corporeal neural tissue has issued from the classical biophysics of mind and brain in homo sapiens, and, in research on non-human subjects precluding self-reports via human language abilities, even correlations have remained substantially elusive. Quantum approaches may offer greater latitude in addressing 
these classical deficiencies, to the extent that at least some latent links formally exist between the qualitative dimensionality and quantitative measurability of canonically conjugate quantum observables, whereas no such formal links are required with reference to the possessed observables of classical physics. Moreover, at least one interpretation of quantum measurement as formulated by von Neumann (1932) casts the measuring agency itself as subjectively conscious per se, in contrast to an absence of any such classical notion.

Quantum generalization of classical biophysics opens up the possibility that relevant brain processes may reach both beneath the scale and beyond the boundaries of discrete neurons and the synaptic connections among those classically cellular "nodes". Quantum-germane structures and dynamics within the brain may include superposed dimeric tubulin conformations in the microtubular cytoskeleton spanning both intraneuronal and interneuronal spaces, ordered water in relation to cytoskeletal proteins, membrane channels and lipids along with their second messenger pipe lines to neuronal interstices, and solitons communicating along cytoskeletal routes between classical and quantum aspects of brain function. Tegmark's (2000) objections to the thermodynamic feasibility of such quantum structures and processes surviving thermal decoherence at biological temperatures entailing orders of magnitude comparable to those within the human skull have been thrown into doubt by the recent work of Gregory Engel's group (Panitchayangkoon et al., 2010), which demonstrated non-trivial quantum computation in photosynthesis. The ubiquity of water, cytoskeletal tubulin, membrane lipids, and second messengers in non-human life suggests that a new biophysics accounting for quantum-generalized processes in living tissue may lead to future predictions about consciousness not only in human beings but also in organisms lacking any semblance of human brain architecture at the level of organized neuronal networks or "higher".

\section{Conclusion}

With reference to quantum approaches to psychopathology, what are the potential ethical applications?

Some powerful contributions of the QPP group (Woolf et al., 2010; Pylkkänen, 2010; Zizzi \& Pregnolato, 2012; Mender, 2013), and especially of the Q-NeMoMa (Quantum Neuron Molecular Mapping) Project pertaining to the European QPP group headed by Massimo Cocchi, Fabio Gabrielli, and Lucio Tonello have raised crucial questions on therapeutic purposes and potential manipulative results on consciousness (consciousness standardization and control, establishment of control systems, push for consumption, weaponization, etc.).

Research done by Cocchi's, Gabrielli's and Tonello's group (Cocchi et al. 2011; Cocchi et al. 2013a; Cocchi et al. 2013b; Cocchi et al. 2015a; Cocchi et al. 2015b; Tonello et al. 2015) aimed at defining the experimental procedures to identify molecular modifications of neurons according to changes in neuronal membrane viscosity (quantum and molecular computation), opens new important perspectives for the treatment of mood disorders (Major Depression and 
Bipolar Disorder), while raising, in the meantime, the issue of finding the most suitable interpretative and applicative ethics.

We think that Aristotle's "virtues", coupled with Spinoza's principle of autonomy, can trace in the best way possible a potential "quantum ethics". Aristotle's "virtues" seem consistent with the super-positional logics of quantum psychology and neurosciences (states that are definable only after having been measured, so they can be assessed only on a case by case basis, according to phronesis, balance, practical reason of the limit). Moreover, and in particular, Anders's (1956) "promethean gap" invalidates (since in the Technological Era the relationship between means and ends is not predictable) the explanatory and applicable force of the general principles underpinning personalist ethics (both deontological and theological): from Kant's formalism to personalist and NeoScholastic ethics, from Weber's (1918) ethics of consequences to responsibility ethics, with the related Jonas' heuristic of fear. So, without general principles, reference can be made to Aristotle's phronesis, considered as the measurement applicable to the decisions to be taken on a case by case basis (regulatory principle for praxis). This must be set in a minimal shared ethical horizon: Spinoza's principle of autonomy (Eth., III, Ax.; IV, P.III), that is to say the common acknowledgement that the more one can represent the "resistance point" against the non-recognition of his "natural" conatus sese conservandi, the more he will be able to increase his power.

\section{References}

Anders, G. (1956). Die Antiquiertheit des Menschen. Munich: Beck.

Bohm, D. (1951). Quantum Theory. Upper Saddle River, NJ: Prentice Hall.

Chalmers, D. (1995). Facing up to the Problem of Consciousness. Journal of Consciousness Studies, 2, 200-219.

Cocchi, M., Tonello, L., Gabrielli, F., \& Castaldo, A. (2013a). Music, Brain, Consciousness. Lugano: Ludes University Press.

Cocchi, M., Gabrielli, F., Tonello, L., Delogu, M., Beghelli, V., Mattioli, M., \& Accorsi, P. A. (2013b). Consciousness from Animals to Humans: Quantum and Molecular Pathways. Quantum Biosystems QPP Group, 5, 19-20.

Cocchi, M., Minuto, C., Tonello, L., \& Tuszynski, J. A. (2015a). Connection between the Linoleic Acid and Psychopathology: A Symmetry-Breaking Phenomenon in the Brain? Open Journal of Depression, 4, 41-52. https://doi.org/10.4236/ojd.2015.44005

Cocchi, M., Tonello, L., \& Gabrielli, F. (2015b). Molecular and Quantum Approach to Psychopathology and Consciousness. Annals of Depression and Anxiety, 2, 1046.

Cocchi, M., Tonello, L., Gabrielli, F., Pregnolato, M., \& Pessa, E. (2011). Quantum Hypothesis on Human and Animal Consciousness: A Concept Embracing Philosophy. Quantitative Molecular Biology and Mathematics. Journal of Consciousness Exploration \& Research, 2, 547-574.

Derrida, J., \& Wills, D. (2002). The Animal That Therefore I Am (More to Follow). Critical Inquiry, 28, 369-418. http://www.jstor.org/stable/1344276 https://doi.org/10.1086/449046

Flanagan, O. (1991). Varieties of Moral Personalities. Ethics and Psychological Realism. Cambridge, MA: Harvard University Press. 
Levine, J. (1983). Materialism and Qualia: The Explanatory Gap. Pacific Philosophical Quarterly, 64, 354-361.

Mender, D. (2013). The Cunning of the Observable: Generalized Fourier Duality, The Hard Problem, and Natural Kinds of Psychopathology. Quantum Biosystems, 5, 43-54.

Panitchayangkoon, G. et al. (2010). Long-Lived Quantum Coherence in Photosynthetic Complexes at Physiological Temperature. Proceedings of the National Academy of Sciences of the United States of America, 107, 12766-12770. https://doi.org/10.1073/pnas.1005484107

Pothos, E. M., \& Busemeyer, J. R. (2009). A Quantum Probability Model Explanation for Violations of "Rational" Decision Making. Proceedings of the Royal Society B, 276, 2171-2178. https://doi.org/10.1098/rspb.2009.0121

Pylkkänen, P. (2010). Implications of Bohmian Quantum Ontology for Psychopathology. NeuroQuantology, 8, 13-28. https://doi.org/10.14704/nq.2010.8.1.265

Tegmark, M. (2000). Importance of Quantum Coherence in Brain Processes. Physical Review E, 61, 4194-4206. https://doi.org/10.1103/PhysRevE.61.4194

Tonello, L., Cocchi, M., Gabrielli, F., \& Tuszynski, J. A. (2015). On the Possible Quantum Role of Serotonin in Consciousness. Journal of Integrative Neuroscience, 14, 295-308. https://doi.org/10.1142/S021963521550017X

von Neumann, J. (1932). Mathematical Foundations of Quantum Mechanics. Princeton, NJ: Princeton University Press.

Weber, M. (1918). Politik als Beruf. Gesammelte Politische Schriften (Muenchen, 1921) (pp. 396-450). Originally a Speech at Munich University, Munich: Duncker \& Humblodt.

Woolf, N., Craddock, T. J. A., Friesen, D. E., \& Tuszynski, J. A. (2010). Impaired Neuroplasticity and Possible Quantum Processing Derailment in Microtubules. NeuroQuantology, 1, 13-28.

Zizzi, P., \& Pregnolato, M. (2012). Quantum Logic of the Unconscious and Schizophrenia. Paradigms Quantum Logic of the Unconscious and Schizophrenia. NeuroQuantology, 10, 566-579. https://doi.org/10.14704/nq.2012.10.3.550

\section{Submit or recommend next manuscript to SCIRP and we will provide best} service for you:

Accepting pre-submission inquiries through Email, Facebook, LinkedIn, Twitter, etc. A wide selection of journals (inclusive of 9 subjects, more than 200 journals)

Providing 24-hour high-quality service

User-friendly online submission system

Fair and swift peer-review system

Efficient typesetting and proofreading procedure

Display of the result of downloads and visits, as well as the number of cited articles

Maximum dissemination of your research work

Submit your manuscript at: http://papersubmission.scirp.org/

Or contact ojd@scirp.org 